Radhika Rastogi, BA

Cleveland Clinic Lerner College of Medicine

of Case Western Reserve University,

Cleveland, $\mathrm{OH}$
Ellen S. Rome MD, MPH

Head, Center for Adolescent Medicine, Department

of General Pediatrics, Cleveland Clinic; Professor,

Cleveland Clinic Lerner College of Medicine of Case

Western Reserve University, Cleveland, $\mathrm{OH}$

\title{
Restrictive eating disorders in previously overweight adolescents and young adults
}

\section{ABSTRACT}

Eating disorders are common in adolescents and young adults, including those with a history of overweight or obesity, and are associated with numerous systemic sequelae. Understanding the differences in presentation between these patients and those who have a more classic anorexia nervosa phenotype is critical to ensuring timely recognition and treatment.

\section{KEY POINTS}

Patients who are overweight or obese are as likely to experience disordered eating as their normal-weight peers.

Patients with a history of premorbid overweight or obesity present differently than their previously healthy weight peers in clinic.

Malnutrition resulting from restrictive eating disorders affects many systems in the body and can occur even in the face of obesity or overweight status.

Few studies have assessed treatment goals and recovery course in previously overweight patients presenting with eating disorders, making it difficult to use evidence-based medicine to manage these cases and assess response to treatment.
Jeannette is a 22-year-old woman who presents with a chief complaint of fatigue. A history reveals that she has lost weight in the past 6 monthsshe used to weigh $220 \mathrm{lb}$ but now weighs 180; her height is 5' 4". She lost weight by reducing her portion sizes, eliminating junk food and anything else "unhealthy," attending a spinning class 4 days a week, and walking at least 10,000 steps every day, as recommended at her well visit a year ago when her body mass index (BMI) was $37.8 \mathrm{~kg} /$ $\mathrm{m}^{2}$. Her BMI is now $30.9 \mathrm{~kg} / \mathrm{m}^{2}$. On examination, her heart rate is 50 beats per minute, and she has mild orthostatic changes in pulse but not in blood pressure. What do you think is going on?

See related editorial, page 172

S ome patients with restrictive eating disor$\checkmark$ ders are hiding in plain sight. Although a patient who starts at $120 \mathrm{lb}$ and loses 40 will look anorectic, someone who starts at $220 \mathrm{lb}$ and comes in weighing 180 may not, despite losing the same amount of weight through the same disordered eating behaviors and putting herself or himself at the same risk of physical harm through starvation.

Patients who are overweight or obese are in a tough position. We want them to lose weight, but we want them to do so safely. Restrictive eating disorders tend to be underrecognized in this group, even though research suggests that these patients may have a greater likelihood of disordered eating habits than those with no history of overweight or obesity. It is therefore important for primary care physicians to consider eating disorders in young patients at a variety of weights. 
This paper discusses the distinguishing features of eating disorders in patients with a history of overweight or obesity, the systemic effects of malnutrition in the body, and general guidelines for medical management.

\section{RISING OBESITY RATES MEET UNREALISTIC NORMS}

In today's body-conscious world, marketers aggressively advertise weight-loss strategies to the public, especially adolescents and young adults. Given that many young people are overweight or obese and that the campaigns tend to focus on the negative effects of excess body fat, disordered eating has been normalized and, in some settings, encouraged. Successful weight loss is praised, even though the methods may be pathologic and involve eating disorder attitudes or behaviors.

It is therefore not surprising that eating disorders are common among children, adolescents, and adults, including those who are overweight or obese.

\section{COMMON AND UNDERRECOGNIZED}

A systematic review of 94 studies published

Heavier patients are as likely to have disordered eating as are their peers with normal weight between 2000 and 2018 reported that the prevalence of eating disorders in the United States was $4.6 \%$, with lower rates in Europe $(2.2 \%)$ and Asia (3.5\%), and higher rates in women $(5.7 \%)$ than in men $(2.2 \%) .{ }^{1}$ The onset of these disorders peaks in adolescence and young adulthood and is associated with poor physical and psychological outcomes. ${ }^{2}$

Although one study reported that a higher percentage of men with eating disorders had a history of premorbid overweight than women, another found no difference by sex..$^{3,4}$

Traditionally, restrictive eating disorders were characterized by low weight or failure to grow appropriately within expected growth curves, maladaptive weight loss strategies, and fear of weight gain. However, these criteria were modified in the fifth edition of the Diagnostic and Statistical Manual of Mental Disorders (DSM-5), demonstrating the evolving clinical understanding of restrictive eating disorders. Of note, the numeric definition of low weight as less than $85 \%$ of median body weight for age has been modified to include patients with less than minimally normal weight. These changes reflect the growing recognition that disordered eating behaviors and associated sequelae can occur at a variety of weights.

In adolescents and young adults with restrictive eating disorders, the prevalence of a history of overweight or obesity ranges from $19.3 \%$ to $36.7 \% .^{3-5}$ These numbers mirror the national prevalence of overweight or obesity, suggesting that patients with higher weight status are as likely to experience disordered eating as their normal-weight peers. ${ }^{4}$

Indeed, overweight adolescents report greater concern about their weight and more body dissatisfaction than their normal-weight peers. ${ }^{6}$ These concerns could lead them to engage in disordered eating behaviors, including bingeing, purging, and diet pill use. ${ }^{6-9}$ There is now a greater recognition of eating disorder diagnoses in young adults who may not meet low-weight criteria but otherwise have classic symptoms.

\section{GREATER WEIGHT SUPPRESSION IN PREVIOUSLY OVERWEIGHT PATIENTS}

Although they present with a higher BMI compared with patients with anorexia nervosa or bulimia nervosa who started out in the normal-weight category, those who started out overweight or obese lose more weight (have greater "weight suppression"), whether the change is measured in kilograms, BMI, or percent body weight. ${ }^{3,4}$ The higher weight at presentation results in a more common diagnosis of atypical anorexia nervosa in these patients. ${ }^{3}$

The amount of weight suppression is a valuable indication of disease severity as well as a prognostic tool. ${ }^{3,4} \mathrm{~A}$ greater percent weight loss has been associated with worse psychological and physical symptoms in patients with anorexia nervosa. ${ }^{10,11}$ A position paper from the Society for Adolescent Health and Medicine has advocated for greater recognition of the clinical utility of percent weight loss and emphasizes the dual roles of weight status as well as rate and relative amount of weight loss. ${ }^{12}$

When the degree of weight suppression based on percent body weight loss is considered, then there is no difference in the likelihood of inpatient treatment, suggesting that weight suppression is a more appropriate met- 


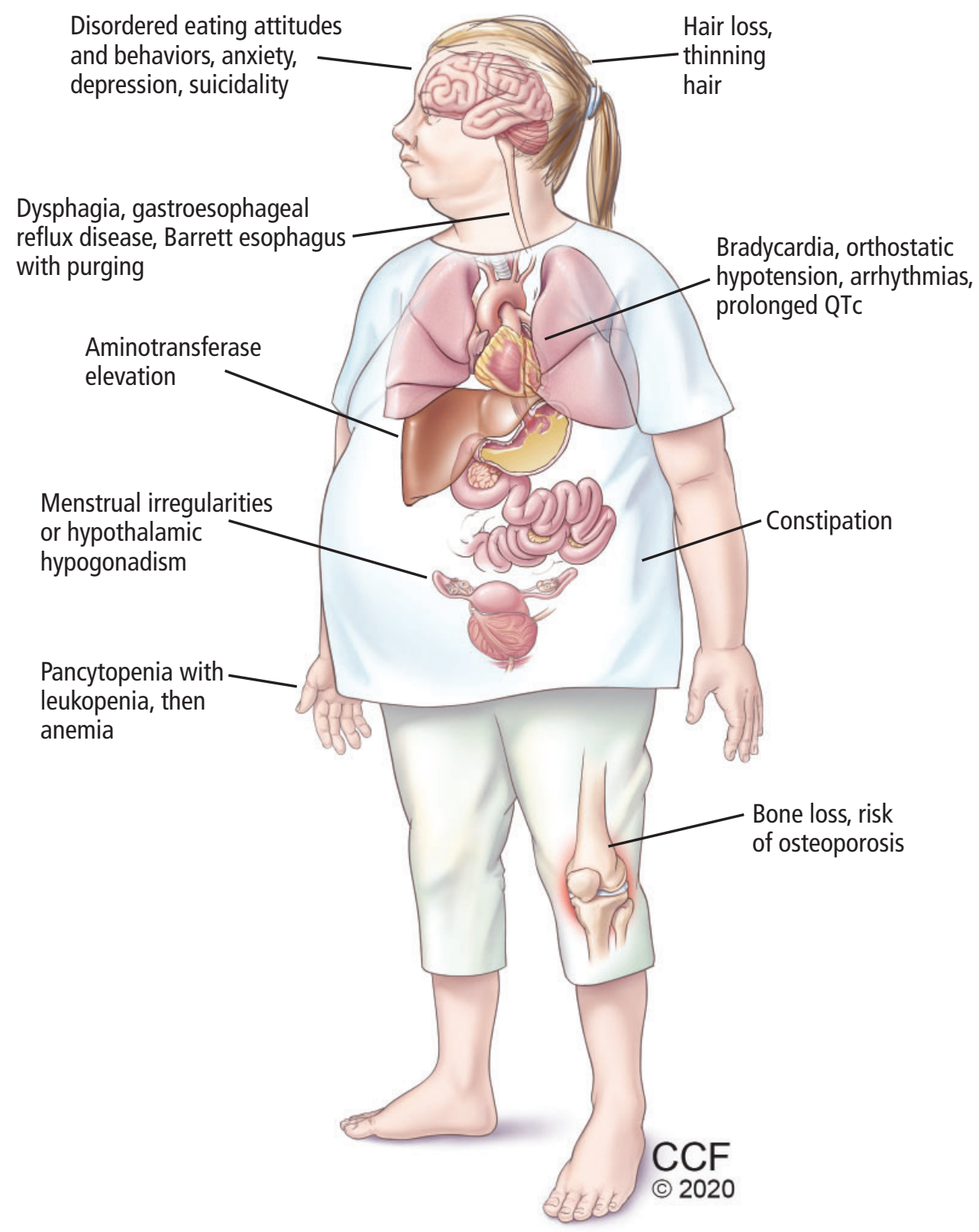

Figure 1. Sequelae and correlates of unhealthy weight loss.

\section{Patients with a history of overweight or obesity present with greater absolute weight loss, but higher BMI}

ric of starvation state than absolute weight status. ${ }^{3}$

Previously overweight adolescents are less likely to receive inpatient treatment despite similar symptoms compared with their previously normal weight peers. ${ }^{3}$ This discrepancy has been attributed to the higher weight at presentation. ${ }^{3}$ This relationship was found to be mediated by weight suppression, supporting the trend toward assessing degree of weight loss rather than focusing primarily on presentation weight when evaluating for eating disorder symptoms. ${ }^{3}$

Comparisons of eating disorder symptom burden based on the Eating Disorder Examination-Questionnaire reveal that previously overweight patients have similar symptoms such as degree of restraint around food and concern about shape or weight. ${ }^{4}$ However, overweight and obese children are more at 
risk than their normal-weight peers of engaging in disordered eating behaviors and exhibiting unhealthy weight loss strategies. ${ }^{13}$

Finally, the duration of illness before presentation may be more variable. Two studies ${ }^{3,4}$ found that the duration of illness is significantly longer in patients with higher premorbid BMIs, whereas another reported that the duration of illness was comparable to that among healthy-weight peers who developed restrictive eating disorders. ${ }^{14}$ These discrepancies may be explained by differences in definitions or small sample sizes.

\section{SYSTEMIC EFFECTS OF MALNUTRITION}

The malnutrition resulting from restrictive eating disorders affects every system in the body and can occur even in the face of obese or overweight status, as seen in the case of Jeannette. Consequences (Figure 1) can be lifelong and include the following:

Cardiovascular manifestations such as bradycardia and hypotension, possibly secondary to increased vagal tone. ${ }^{15,16}$ Consistent hypotension may cause decreased left ventricular mass, resulting in decreased cardiac output. ${ }^{15}$

Recognize Additionally, abnormal electrolyte levels and eating disorders as readily in a patient who shifts from 220 to $180 \mathrm{lb}$ as in a patient who went from 120 to $80 \mathrm{lb}$ vagal tone can contribute to arrhythmias such as prolonged QTc interval. ${ }^{15,16}$

Gastrointestinal effects include gastroparesis and constipation from slow colonic transit. ${ }^{15,16}$ In an extremely underweight patient, loss of fat that normally cushions the superior mesenteric artery (SMA) can result in SMA syndrome, in which the duodenum is pinched between the SMA and the aorta and consequently dilates. Dysphagia from weakening of pharyngeal muscles can hinder refeeding and result in aspiration. Patients can also have aminotransferase elevations due to liver cell apoptosis. ${ }^{15,16}$

Hematologic effects include pancytopenias due to a hypoplastic marrow with fat deposition within the marrow space. ${ }^{15-18}$ Despite the neutropenia, patients with eating disorders are not at higher risk of infections. However, impaired inflammatory responses may result in slower recognition of an infection. ${ }^{15,16}$

Musculoskeletal effects. Patients with eating disorders are at risk of losing both muscle and bone mass. Bones have both altered structure and decreased strength. ${ }^{15,16,18}$ Contributors to weakened bone include derangements in the hypothalamic-pituitary-adrenal axis and the hypothalamic-pituitary-gonadal axis, as well as thyroid dysfunction. Adolescent girls with anorexia were found to have increased fracture risk even in the absence of decreased bone mineral density as measured by dual energy $\mathrm{x}$-ray absorptiometry, suggesting that loss of mass incompletely explains the bone sequelae of eating disorders. ${ }^{18}$

Hormone disturbances include low insulin-like growth factor 1 and estrogen levels, leading to decreased bone deposition, increased resorption, osteopenia, and osteoporosis. ${ }^{15}$ In addition, there may be impaired thyroid response to low thyroid hormone levels, and high cortisol levels with slower clearance may result in longer cortisol half-lives. ${ }^{15,16}$

Menstrual disturbances such as oligomenorrhea and amenorrhea can occur due to dysregulation of the hypothalamic-pituitarygonadal axis, resulting in low estrogen status. ${ }^{19}$ There are similar reductions in testosterone levels for boys. ${ }^{15}$

The consequences for adolescents are tremendous, as $40 \%$ to $60 \%$ of bone density is deposited during early adolescence in girls and late adolescence in boys. Further, hypoestrogenism in adolescence may also be associated with earlier risk of cardiovascular disease. ${ }^{20-22}$ Thus, addressing amenorrhea and hypothalamic hypogonadism is a well-established goal of eating disorder treatment. ${ }^{23,24}$

Death. People with anorexia nervosa have a mortality rate up to 6 times higher than their peers, which is among the highest in psychiatric illnesses. ${ }^{25-27}$ Although the mortality rate associated with bulimia nervosa is lower, these patients have a high rate of suicide. ${ }^{28-31}$

\section{MANAGING EATING DISORDERS IN PREVIOUSLY OVERWEIGHT PATIENTS}

Although many overweight and obese people have eating disorders, most research is limited to underweight patients only. ${ }^{23,24,32}$ Thus, there are few data to guide management of weight loss-related sequelae in previously overweight patients presenting with eating disorders.

In general, body fat mass, amount of relative weight loss, and appropriate hormonal 
levels and interactions contribute to normal menstrual function. ${ }^{33,34}$ Disruption of the hypothalamic-pituitary-ovarian axis by weight loss may be mediated by changes in gonadotropin-releasing hormone $(\mathrm{GnRH})$ release. Typical GnRH function is pulsatile and relies on leptin and insulin-like growth factor 1 (IGF-1) signaling. ${ }^{35-37}$ Fat mass contributes to normal production of both leptin and IGF-1, and therefore loss of fat mass can disrupt the hormonal signaling underlying normal menstruation. . $2,38,39$

Previously overweight patients follow a different course than their previously healthyweight peers in menses recovery. In one study, amenorrheic patients with a history of overweight or obesity resumed menses at a higher weight but with similar amounts of absolute gain in weight. ${ }^{5}$ Additionally, the likelihood of menses resumption decreases with greater weight suppression and increases with greater weight gain during treatment in both groups. ${ }^{5}$ This finding suggests that weight goals associated with resumption of menses may need to be higher for patients with a history of overweight.

Weight restoration may therefore have different effects on adolescents who had different baseline hormonal production, given different fat mass before the onset of illness. The difference in patterns of menses resumption highlights the need to identify physiologic alterations that may differ between previously overweight and previously normal-weight teens at the onset of eating disorders.

That only one study has compared outcomes between adolescents and young adults with eating disorders by premorbid weight status shows the understudied nature of this comparably high-risk population. While there is greater recognition of the differences in the population, there remains a paucity of studies on treatment goals and recovery course, making it difficult to use evidence-based medicine to assess response to treatment.

Until definitive guidelines are published, the primary care physician can use the following general treatment advice:

Encourage healthy forms of weight loss while establishing minimum requirements for nutrition, including protein, fat, carbohydrates, calcium, and vitamin D. Overweight or obese individuals who wish to pursue a ke- togenic or "clean eating" diet should consult with both their primary care clinician and a registered dietician who is experienced in treating patients with eating disorders and obesity to avoid electrolyte imbalances and other medical complications of starvation. If patients are younger than 26 years, recommend adequate fat intake- 50 to $90 \mathrm{~g}$ a daybecause myelination is still occurring in the brain. The primary care clinician, in concert with the dietitian, can help tailor a meal plan to an individual's needs, preventing "hardwiring" of eating disorder behaviors while ensuring adequate intake of essential nutrients.

Monitor for sequelae of caloric energy restriction, including bradycardia, orthostatic hypotension, amenorrhea and oligomenorrhea.

Be vigilant for pathologic weight loss strategies in overweight as well as underweight patients: recognize eating disorders as readily in the patient who shifts from 220 to $180 \mathrm{lb}$ as one would recognize if the patient went from 120 to 80 .

\section{CASE FOLLOW-UP}

Jeannette returns after 1 month, having seen the dietitian and increased her intake to 1,800 kcal per day from her previous 400 to $600 \mathrm{kcal}$ per day. Her weight is now $185 \mathrm{lb}$. Her heart rate has increased to the 60s, but she has ongoing amenorrhea. Her luteinizing hormone level is $2.0 \mathrm{mU} /$ $\mathrm{mL}$ and her follicle-stimulating hormone level is $2.6 \mathrm{mU} / \mathrm{mL}$. These values are on the low-normal side, consistent with functional hypothalamic amenorrhea, in which there is suppression of gonadotropins that is centrally mediated through the release of $\mathrm{G} n \mathrm{RH}$ from the hypothalamus. Her thyroid-stimulating hormone level is normal, and her white blood cell count has normalized (she was previously mildly leukopenic).

\section{TAKE-HOME POINTS}

Restrictive eating disorders are common among adolescents, including those with a history of overweight or obesity.

These patients present with greater weight loss from a higher starting weight but have comparable eating disorder symptoms that may go unrecognized for a longer time and result in suboptimal access to care. 
Differences in presentation and recovery highlight the need for earlier detection of pathologic behaviors as well as more personalized intervention strategies.

The paucity of evidence on treatment goals makes it challenging to treat previously overweight patients. Early recognition and prevention of significant morbidity is key as further investigations on differences in recovery course are conducted.

Markers of recovery such as resumption of menses suggest that previously overweight patients resume physiologic function at a higher weight and with comparable amount of weight gain as their previously normal weight peers. The differences in weight status at menses resumption reflect the complex neurohormonal mechanisms that underlie functional hypothalamic amenorrhea.

The differences in presentation characteristics underscore the need to emphasize percent weight loss alone rather than extreme low body weight as a metric for eating disorder pathology. How a patient loses weight, as well as the accompanying disordered eating attitudes and behaviors, can illuminate pathology in the obese patient as well as in the severely underweight individual. If the emphasis remains on extreme low body weight as the marker of illness, other findings of starvation - including low heart rate and lower blood pressure in the obese, overweight, and even normal-weight patient who is in the same state of starvation-will continue to be unrecognized. ${ }^{3}$

\section{REFERENCES}

1. Galmiche M, Déchelotte P, Lambert G, Tavolacci MP. Prevalence of eating disorders over the 2000-2018 period: a systematic literature review. Am J Clin Nutr 2019; 109(5):1402-1413. doi:10.1093/ajen/nqy342

2. Nagl M, Jacobi C, Paul M, et al. Prevalence, incidence, and natural course of anorexia and bulimia nervosa among adolescents and young adults. Eur Child Adolesc Psychiatry 2016; 25(8):903-918. doi:10.1007/s00787-015-0808-z

3. Kennedy GA, Forman SF, Woods ER, et al. History of overweight/ obesity as predictor of care received at 1-year follow-up in adolescents with anorexia nervosa or atypical anorexia nervosa. J Adolesc Health 2017; 60(6):674-679. doi:10.1016/j.jadohealth.2017.01.001

4. Lebow J, Sim LA, Kransdorf LN. Prevalence of a history of overweight and obesity in adolescents with restrictive eating disorders. J Adolesc Health 2015; 56(1):19-24. doi:10.1016/j.jadohealth.2014.06.005

5. Seetharaman S, Golden NH, Halpern-Felsher B, Peebles R, Payne A, Carlson JL. Effect of a prior history of overweight on return of menses in adolescents with eating disorders. J Adolesc Health. 2017; 60(4):469-471. doi:10.1016/j.jadohealth.2016.10.019

6. Calzo JP, Soneville KR, Haines J, Blood EA, Field AE, Auston SB. The development of associations among body mass index, body dissatisfaction, and weight and shape concern in adolescent boys and girls. J Adolesc Health 2012; 51(5):517-523. doi:10.1016/j.jadohealth.2011.10.252

7. Cromley T, Knatz S, Rockwell R, Neumark-Sztainer D, Story M, Boutelle K. Relationships between body satisfaction and psychological functioning and weight-related cognitions and behaviors in overweight adolescents. J Adolesc Health 2012; 50(6):651-653. doi:10.1016/j.jadohealth.2011.10.252

8. Neumark-Sztainer D, Wall M, Guo J, Story M, Haines J, Eisenberg M. Obesity, disordered eating, and eating disorders in a longitudinal study of adolescents: how do dieters fare 5 years later? J Am Diet Assoc 2006; 106(4):559-568. doi:10.1016/j.jada.2006.01.003

9. Neumark-Sztainer D, Wall M, Story M, Sherwood NE. Five-year longitudinal predictive factors for disordered eating in a population-based sample of overweight adolescents: implications for prevention and treatment. Int J Eat Disord 2009; 42(7):664-672. doi:10.1002/eat.20733

10. Berner LA, Shaw JA, Witt AA, Lowe MR. The relation of weight suppression and body mass index to symptomatology and treatment response in anorexia nervosa. J Abnorm Psychol 2013; 122(3):694708. doi:10.1037/a0033930
11. Peebles R, Hardy KK, Wilson JL, Lock JD. Are diagnostic criteria for eating disorders markers of medical severity? Pediatrics 2010; 125(5):e1193-e1201. doi:10.1542/peds.2008-1777

12. Society for Adolescent Health and Medicine; Golden NH, Katzman DK, Sawyer SM, et al. Position paper of the Society for Adolescent Health and Medicine: medical management of restrictive eating disorders in adolescents and young adults. J Adolesc Health 2015; 56(1):121-125. doi:10.1016/j.jadohealth.2014.10.259

13. Cena H, Stanford FC, Ochner L, et al. Association of a history of childhood-onset obesity and dieting with eating disorders. Eat Disord 2017; 25(3):216-229. doi:10.1080/10640266.2017.1279905

14. Witt AA, Berkowitz SA, Gillberg C, Lowe MR, Råstam M, Wentz E. Weight suppression and body mass index interact to predict long-term weight outcomes in adolescent-onset anorexia nervosa. J Consult Clin Psychol 2014; 82(6):1207-1211. doi:10.1037/a0037484

15. Mehler PS, Brown C. Anorexia nervosa-medical complications. J Eat Disord 2015;3:11. doi:10.1186/s40337-015-0040-8

16. Westmoreland P, Krantz MJ, Mehler PS. Medical complications of anorexia nervosa and bulimia. Am J Med 2016; 129(1):30-37. doi:10.1016/j.amjmed.2015.06.031

17. Ecklund K, Vajapeyam S, Feldman HA, et al. Bone marrow changes in adolescent girls with anorexia nervosa. J Bone Miner Res 2010; 25(2):298-304. doi:10.1359/jbmr.090805

18. Donaldson AA, Gordon CM. Skeletal complications of eating disorders. Metabolism 2015; 64(9):943-951. doi:10.1016/j.metabol.2015.06.007

19. Allaway HC, Southmayd EA, De Souza MJ. The physiology of functional hypothalamic amenorrhea associated with energy deficiency in exercising women and in women with anorexia nervosa. Horm Mol Biol Clin Investig 2016; 25(2):91-119. doi:10.1515/hmbci-2015-0053

20. Solomon CG, Hu FB, Dunaif A, et al. Menstrual cycle irregularity and risk for future cardiovascular disease. J Clin Endocrinol Metab 2002; 87(5):2013-2017. doi:10.1210/jcem.87.5.8471

21. Weitzmann MN, Pacifici R. Estrogen deficiency and bone loss: an inflammatory tale. J Clin Invest 2006; 116(5):1186-1194. doi: $10.1172 / \mathrm{JCl} 28550$

22. Meczekalski B, Podfigurna-Stopa A, Genazzani AR. Hypoestrogenism in young women and its influence on bone mass density. Gynecol Endocrinol 2010; 26(9):652-657. doi:10.3109/09513590.2010.486452

23. Dempfle A, Herpertz-Dahlmann B, Timmesfeld N, et al. Predictors of the resumption of menses in adolescent anorexia nervosa. BMC Psychiatry 2013; 13:308. doi:10.1186/1471-244X-13-308

24. Golden NH, Jacobson MS, Schebendach J, Solanto MV, Hertz SM, 
Shenker IR. Resumption of menses in anorexia nervosa. Arch Pediatr Adolesc Med 1997; 151(1):16-21.

doi:10.1001/archpedi.1997.02170380020003

25. Sigel E. Eating disorders. Adolescent Med State Art Rev 2008; 19(3):547-572. pmid:19227391

26. Herpertz-Dahlmann B. Adolescent eating disorders: definitions, symptomatology, epidemiology and comorbidity. Child Adolesc Psychiatr Clin N Am 2009; 18(1):31-47. doi:10.1016/j.chc.2008.07.005

27. Franko DL, Keshaviah A, Eddy KT, et al. A longitudinal investigation of mortality in anorexia nervosa and bulimia nervosa. Am J Psychiatry 2013; 170(8):917-925. doi:10.1176/appi.ajp.2013.12070868

28. Hoste RR, Labuschagne Z, Le Grange D. Adolescent bulimia nervosa. Curr Psychiatry Rep 2012; 14(4):391-397. doi:10.1007/s11920-012-0280-0

29. Swanson SA, Crow SJ, Le Grange D, Swendsen J, Merikangas KR. Prevalence and correlates of eating disorders in adolescents. Results from the national comorbidity survey replication adolescent supplement. Arch Gen Psychiatry 2011; 68(7):714-723. doi:10.1001/archgenpsychiatry.2011.22

30. Sim LA, McAlpine DE, Grothe KB, Himes SM, Cockerill RG, Clark MM. Identification and treatment of eating disorders in the primary care setting. Mayo Clin Proc 2010; 85(8):746-751. doi:10.4065/mcp.2010.0070

31. Fichter MM, Quadflieg N. Twelve-year course and outcome of bulimia nervosa. Psychol Med 2004; 34(8):1395-1406. doi:10.1017/s0033291704002673

32. Tokatly Latzer I, Kidron-Levy H, Stein D, et al. Predicting menstrual recovery in adolescents with anorexia nervosa using body fat percent estimated by bioimpedance analysis. J Adolesc Health 2019; 64(4):454-460. doi:10.1016/j.jadohealth.2018.10.008

33. Mitan LA. Menstrual dysfunction in anorexia nervosa. J Pediatr Adolesc Gynecol 2004; 17(2):81-85. doi:10.1016/j.jpag.2004.01.003

34. Vyver E, Steinegger C, Katzman DK. Eating disorders and menstrual dysfunction in adolescents. Ann N Y Acad Sci 2008; 1135:253-264. doi:10.1196/annals.1429.013

35. Meczekalski B, Katulski K, Czyzyk A, Podfigurna-Stopa A, Maciejewska-Jeske M. Functional hypothalamic amenorrhea and its influence on women's health. J Endocrinol Invest 2014; 37(11):1049-1056. doi:10.1007/s40618-014-0169-3

36. Daftary SS, Gore AC. IGF-1 in the brain as a regulator of reproductive neuroendocrine function. Exp Biol Med (Maywood) 2005; 230(5):292-306. doi:10.1177/153537020523000503

37. Kelesidis T, Kelesidis I, Chou S, Mantzoros CS. Narrative review: the role of leptin in human physiology: emerging clinical applications. Ann Intern Med 2010; 152(2):93-100. doi:10.7326/0003-4819-152-2-201001190-00008

38. Adriana S, Gambera A, Specchia C, Pellegrini C, Falsetti L, Sartori E. Leptin in functional hypothalamic amenorrhoea. Hum Reprod 2002; 17(8):2043-2048. doi:10.1093/humrep/17.8.2043

39. Miller KK, Grinspoon S, Gleysteen S, et al. Preservation of neuroendocrine control of reproductive function despite severe undernutrition. J Clin Endocrinol Metab 2004; 89(9):4434-4438. doi:10.1210/jc.2004-0720

Address: Radhika Rastogi, BA, Cleveland Clinic Lerner College of Medicine of Case Western Reserve University, 9501 Euclid Avenue, EC10, Cleveland, $\mathrm{OH} 44195 ;$ rr2710@gmail.com 